Enfermagem Brasil 2018;17(5):452-9

\title{
ARTIGO ORIGINAL \\ Adaptação transcultural da Spiritual Needs Assessment Scale para o idioma português
}

\author{
Ewerton Naves Dias*, José Luís Pais-Ribeiro** \\ *Doutorando em Psicologia pela Faculdade de Psicologia e Ciências da Educação da \\ Universidade do Porto, Portugal, ${ }^{* * D o c e n t e}$ da Universidade de Mogi das Cruzes, São \\ Paulo, ${ }^{\star \star}$ Docente da Faculdade de Psicologia e de Ciências da Educação da Universidade do \\ Porto, Porto, Portugal
}

Recebido 3 de novembro de 2017; aceito em 23 de abril de 2018.

Endereço para correspondência: Ewerton Naves Dias, Rua Alfredo Allen, Faculdade de Psicologia da Universidade do Porto, 4200-135 Porto, Portugal, E-mail: ewertonnaves@gmail.com; José Luís Pais-Ribeiro: jlp@fpce.up.pt

\begin{abstract}
Resumo
Crenças espirituais e religiosas têm sido apontadas como importantes componentes de qualidade de vida, especialmente para aquelas pessoas gravemente enfermas. Entretanto, na prática clínica os profissionais da saúde têm encontrado dificuldades para abordar os aspectos espirituais dos pacientes. Para agravar essa situação, ainda são poucos os instrumentos para medir as necessidades espirituais, principalmente em outro idioma que não seja o inglês. Diante deste contexto, o objetivo deste estudo foi adaptar transculturalmente a Spiritual Needs Assessment Scale para a língua portuguesa. Trata-se de um estudo de adaptação transcultural realizado de acordo com as etapas proposta por Beaton et al. A Spiritual Needs Assessment Scale é composta por 28 itens do tipo Likert e foi construída com o propósito de avaliar as necessidades espirituais dos pacientes. A versão final da escala adaptada recebeu o nome de "Escala de avaliação de necessidades espirituais". Espera-se que este estudo possa contribuir futuramente para a prática clínica dos profissionais de saúde e também para os estudos sobre este tema.
\end{abstract}

Palavras-chave: adaptação transcultural, espiritualidade, psicometria.

\begin{abstract}
Cross-cultural adaptation of the Spiritual Needs Assessment Scale to the Portuguese language

Spiritual and religious beliefs have been identified as important components of quality of life and especially in critically ill patients. However, in clinical practice, health professionals have found it difficult to approach the spiritual aspects of patients. To exacerbate this situation, there are still few instruments to measure the patients' spiritual needs, especially in a language other than English. The purpose of this study was to transculturally adapt the "Spiritual Needs Assessment Scale" to the Portuguese language. This is a cross-cultural adaptation study carried out according to the steps proposed by Beaton et al. The "Spiritual Needs Assessment Scale" was constructed from a broad literature review in order to assess the spiritual needs of the patients; the scale is composed of 28 Likert-type items. After the necessary adjustments, the final version of the scale adapted for Portuguese language was called "Scale of Assessment of Spiritual Needs". It is expected that this study may contribute to the clinical practice of health professionals and also to future studies on this topic.
\end{abstract}

Key-words: cross-cultural adaptation, spirituality, psychometry.

\section{Resumen}

\section{Adaptación transcultural de la Spiritual Needs Assessment Scale al portugués}

Las creencias espirituales y religiosas han sido señaladas como importantes componentes de calidad de vida, especialmente para aquellas personas gravemente enfermas. Sin embargo, en la práctica clínica los profesionales de la salud han encontrado dificultades para abordar los aspectos espirituales de los pacientes. Para agravar esa situación todavía son pocos los instrumentos para medir las necesidades espirituales, principalmente en otro idioma que no sea el inglés. En este contexto, el objetivo de este estudio fue adaptar culturalmente a Spiritual Needs Assessment Scale al portugués. Se trata de un estudio de adaptación transcultural 
realizado de acuerdo con las etapas propuestas por Beaton et al. La Spiritual Needs Assessment Scale está compuesta por 28 ítems del tipo Likert y fue construida con el propósito de evaluar las necesidades espirituales de los pacientes. La versión final de la escala adaptada recibió el nombre de "Escala de Evaluación de Necesidades Espiritual". Se espera que este estudio pueda contribuir en el futuro a la práctica clínica de los profesionales de la salud y también a los estudios sobre este tema.

Palabras-clave: adaptación transcultural, espiritualidad, psicometría.

Introdução

No que diz respeito ao senso comum é habitual às pessoas conceituarem a religião, religiosidade e a espiritualidade como fenômenos sinônimos, entretanto, apesar de estarem relacionados, estes temas possuem significados e características próprias. Tavares et al. comentam que os conceitos de religião, religiosidade e espiritualidade vem sendo utilizados repetidamente de forma equivalente, acarretando equívocos que podem desviá-los de seus reais significados [1]. Dessa forma, no contexto científico, uma primeira e importante medida a ser tomada ao investigar esses temas é a de definir os seus aspectos teóricos e conceituais, no sentido de elucidar as particularidades que envolvem cada um deles. Assim, são apresentados a seguir os principais aspectos conceituais adotados neste estudo sobre cada um destes construtos.

Quanto à definição do conceito de religião é importante mencionar que o mesmo já foi definido de muitas formas, e que muitas dessas definições se sobrepõem. De forma geral, a religião pode ser entendida como um sistema complexo de crenças (sobre a realidade, a pessoa humana e a moralidade) que regulam a vida (influenciam o modo como vivemos), que são expressas em certos tipos de rituais e práticas, e que se baseiam, em grande parte, na crença em uma realidade sagrada e transcendente (invisível) [2]. As religiões podem ser entendidas ainda como "instituições organizadas em torno da ideia de espírito e referem-se a sistemas de crenças e cultos que as pessoas herdam ou adotam, e que entendem ser meios que conduzem à felicidade e à satisfação. O propósito da religião é prover uma estrutura onde se possa desenvolver uma consciência espiritual" [3].

Em contraposição à religião, a noção de religiosidade é vista como algo mais pessoal, ou seja, menos atrelado a instituições religiosas e a comportamentos ritualizados ou a doutrinas religiosas específicas. O filósofo e sociólogo alemão Georg Simmel define a religiosidade como um "estado" ou "necessidade" interna, assim como um conjunto de crenças ou conhecimentos que a tradição oferece na tentativa de satisfazer tal necessidade [4]. Portanto, a religiosidade incluiria as crenças pessoais, tais como a crença em um Deus ou poder superior, assim como as crenças e práticas institucionais, como a pertença a denominações religiosas, a frequência a cultos e o compromisso com um sistema doutrinário de uma Igreja ou religião organizada [5]. De acordo ainda com estudiosos sobre este assunto, à religiosidade pode ser entendida como uma extensão na qual o indivíduo acredita, segue e pratica uma religião. Esta pode ser organizacional (participação no templo religioso), não organizacional (rezar, ler livros, assistir programas religiosos na televisão) e intrínseca (relacionada ao quanto à pessoa se considera religiosa) $[5,6]$.

Quanto à espiritualidade, o seu uso destacado da religião e religiosidade é ainda um acontecimento recente, a secularização e a desilusão com as instituições religiosas no Ocidente fizeram com que a noção de espiritualidade ganhasse sentido e conotação diferente da de religião e religiosidade. Nesse sentido, a religiosidade e a espiritualidade, embora constituam campos semânticos sobrepostos, passaram a ser diferenciadas. Em termos conceituais, a espiritualidade tem sido definida como um constructo com dimensão mais pessoal e existencial, como a crença e/ou relação com Deus ou um poder superior [7]. Em outras palavras, a espiritualidade pode ser definida também como um sistema de crenças que enfoca elementos que transcendem o tangível, como uma propensão humana para encontrar um significado para a vida, um sentido de conexão com algo maior que si próprio, que pode ou não incluir uma participação religiosa formal. Tal crença pode mobilizar energias e iniciativas extremamente positivas, com potencial ilimitado para melhorar a qualidade de vida da pessoa [8].

No que tange a área da saúde as crenças espirituais e religiosas têm sido apresentadas como importantes componentes de qualidade de vida em todas as sociedades. Profissionais de saúde, pesquisadores e a população em geral têm confirmado a importância da dimensão religiosa e espiritual para a saúde física e mental das pessoas $[9,10]$. Evidências 
têm demonstrado que as pessoas com espiritualidade bem desenvolvida tendem a adoecer menos, a ter hábitos de vida mais saudáveis e, que quando adoecem, desenvolvem menos depressão e recuperam-se mais rapidamente da enfermidade. Estudos recentes apontam também que a espiritualidade e a religiosidade têm sido associadas a melhor função imunológica, menores taxas de mortalidade devido ao câncer e a doenças cardíacas, menores níveis de colesterol e pressão arterial, a comportamentos saudáveis como (fumar menos, praticar mais exercícios e ter melhor qualidade de sono), redução das taxas de suicídio, menor ansiedade, menor uso de substâncias proibidas, menor depressão e recuperação mais rápida da mesma, maior bem-estar, maior apoio social, maior estabilidade e satisfação conjugal, esperança, otimismo e mais propósito e significado para a vida [10,11].

Estudos citam ainda a espiritualidade como um fator que auxilia no cuidado de pacientes com câncer ou doenças terminais, em seus diferentes estágios de evolução. Estas investigações têm como principal foco de estudo pacientes com essas características, pois, devido à situação patológica em que se encontram, são mais suscetíveis ao apego às suas crenças religiosas e espirituais como modo de enfrentamento ou coping religioso e, por conseguinte, permite analisar o quanto esse tipo de recurso auxilia no processo de cura ou tratamento. Vale ainda mencionar que, estes estudos citam a espiritualidade como uma dimensão importante da qualidade de vida [9]. De modo geral, nota-se por meio das pesquisas que há tendência à correlação entre a religiosidade/ espiritualidade e a saúde física e saúde mental. No entanto, alguns pesquisadores alertam que ainda são necessários novos estudos com provas e correlações mais robustas sobre essa questão [12].

Já na prática clínica, embora os profissionais reconheçam o cuidado à dimensão espiritual como uma ferramenta importante para a saúde e conforto dos pacientes, encontram dificuldades em abordá-la, pois na maior parte das vezes não receberam durante a formação acadêmica preparação para lidar com esse fenômeno. Muitos deles entendem ainda que as necessidades espirituais dos pacientes não são de sua competência, e que estas devem ser abordadas por sacerdotes, pastores ou pessoas religiosas [13]. Diante dessa realidade, o paciente é o principal prejudicado, pois deixa de receber cuidado integral as suas necessidades, neste caso em especial a sua espiritualidade.

Outra problemática encontrada pelos estudiosos nessa área do conhecimento se refere ao número limitado de instrumentos disponíveis na literatura, sobretudo, para medir as necessidades espirituais das pessoas, na atualidade são poucas as escalas dessa natureza, principalmente quando se trata de escalas em outro idioma que não seja o inglês. Estudo de revisão sistemática em diversas bases de dados para coletar informações sobre as escalas e instrumentos destinados a medir a espiritualidade e a religiosidade já traduzidas para o Português, encontrou 20 instrumentos para medir a espiritualidade e/ou religiosidade disponível no idioma Português, sendo a maioria destes instrumentos traduzidos, adaptados ou desenvolvidos no Brasil e com boa consistência interna. Os autores alertam, ainda, que poucos instrumentos foram totalmente avaliados em relação às qualidades psicométricas, ou quanto 0 procedimento de teste-reteste ou confirmação da validade concorrente e divergente. Os autores recomendam que outros estudos de validação são necessários, a fim de avaliar plenamente estes instrumentos de língua portuguesa em uma gama de diferentes amostras [14].

Nota-se, que a maior parte das escalas de espiritualidade disponível na literatura avalia o índice de religiosidade e espiritualidade das pessoas, mas não se essas pessoas necessitam de suporte religioso e/ou espiritual. Existe uma evidente carência de instrumentos no que tange a avaliação das necessidades espirituais, o que acaba por prejudicar a produção de novos estudos e, consequentemente, o desenvolvimento e evolução teórica/conceitual sobre este fenômeno. O número limitado de instrumentos adaptados e validados no que tange a avaliação das necessidades espirituais dos pacientes, além de dificultar o diagnóstico, pode também interferir, consequentemente, na definição das condutas terapêuticas e na elaboração dos planos de intervenção, comprometendo dessa forma a eficácia e a eficiência dos tratamentos oferecidos.

Fica evidente diante desses aspectos a importância de estudos como este que envolvam a construção ou adaptação e validação de escalas específicas para avaliar as necessidades espirituais das pessoas, pois a compreensão do indivíduo em sua essência, como um ser que possui necessidades no âmbito biológico, psicológico, social e espiritual remete a reflexões relacionadas à influência da espiritualidade nas respostas humanas aos agravos à saúde que precisam ser acolhidas para o atendimento dessas necessidades em sua 
plenitude [15]. Diante do que foi exposto este estudo teve como objetivo realizar a adaptação transcultural da Spiritual Needs Assessment Scale $[16,17]$ para a língua portuguesa.

Material e métodos

Trata-se de um estudo de adaptação cultural da Spiritual Needs Assessment Scale realizado de acordo com as etapas propostas na investigação, para a tradução de questionários de uma língua e cultura para outra língua e cultura diferente [18,19]. A International Tests Comission chama a atenção, em especial, para a importância de alguns aspetos, nomeadamente para: efeitos das diferenças culturais, das diferenças linguísticas e gramaticais, se a linguagem é apropriada para a população para a qual está sendo adaptada, se as técnicas de testagem o formato de resposta dos itens, convenções e procedimentos são familiares para a população a que se destina, que deverão manter um julgamento sistemático que suporte a evidência linguística e psicológica de modo a aprimorar a precisão do processo de adaptação, e deverão apresentar evidência da equivalência entre as diversas versões linguísticas [19].

Neste contexto a validade de conteúdo assume uma centralidade decisiva. Ela pode ser definida como o grau em conteúdo do questionário é congruente com os propósitos da avaliação. Esta inclui quatro elementos sobre a qualidade do instrumento: de?nição do domínio, representação do domínio, relevância do domínio, e adequação do processo de desenvolvimento do questionário, ela deriva da análise por especialistas [20]. A validade de conteúdo se refere ao quanto o teste pode ser uma amostra representativa dos comportamentos que são a expressão do traço latente em questão, ou em outras palavras se os itens dos testes se constituem uma amostra representativa do universo de itens do construto estudado [21].

No presente estudo seguimos o guião proposto por Beaton et al. [18]. A autorização para a tradução da escala para a língua portuguesa foi obtida por meio de contato com os autores do referido instrumento. A Spiritual Needs Assessment Scale foi concebida por Galek et al. $[16,17]$ para avaliar as necessidades espirituais dos pacientes. A partir de extensa revisão de literatura nas bases de dados PsycINFO (American Psychological Association) e Medline (National Library of Medicine), sobre o termo de pesquisa necessidades espirituais, os autores realizaram uma análise temática da literatura teórica e empírica que revelou inicialmente 339 itens que refletiam as preocupações do paciente acerca da espiritualidade.

A partir dos itens iniciais, foi elaborada uma escala tipo likert com 28 itens, com pontuações variando de ( $1=$ Nunca) a ( $4=$ Com muita frequência). O escore total é obtido pela soma das pontuações dos 28 itens que podem variar de 28 a 112 pontos. Escores maiores refletem maiores necessidades espirituais. Em outro estudo, os autores realizaram a estrutura fatorial da escala nas respostas de 683 indivíduos. Neste estudo quatro itens da escala original foram excluídos por terem recebidos pontuações baixas durante o teste preliminar. Os resultados com a versão de 24 itens forneceram forte apoio para quatro das seis dimensões de necessidades espirituais proposta pelos autores [17]. Para o presente estudo de adaptação transcultural, utilizou-se por sugestão dos autores da versão original a escala com 28 itens.

Etapas do estudo

Inicialmente realizaram-se duas traduções literais da escala da língua inglesa para a língua portuguesa por dois tradutores independentes bilíngues que tinham como língua materna o português. A seguir as duas primeiras traduções da escala para o português brasileiro foram então comparadas e analisadas pelos autores deste estudo resultando na tradução consensual do referido instrumento.

Após a tradução consensual foram convidados cinco professores de inglês que tiveram a incumbência de realizar a avaliação semântica e idiomática da tradução consensual. Para o registro das avaliações dos professores utilizou-se um instrumento próprio para essa finalidade. Após a avaliação semântica e idiomática e dos ajustes necessários, a nova versão da escala foi traduzida de volta para o inglês por dois novos tradutores bilíngues, para confirmar se a versão traduzida refletia a escala original.

Na próxima etapa foram identificados e convidados pesquisadores com conhecimento e publicações sobre o tema religiosidade e espiritualidade para participarem da análise da equivalência cultural e conceitual da Spiritual Needs Assessment Scale. Os pesquisadores foram identificados por meio do currículo lattes e por sua produção científica ligada a temática deste estudo. Para composição do comitê de juízes especialistas atentou-se para os seguintes critérios de inclusão: ser profissional da área da saúde com doutoramento; ter domínio da 
língua inglesa e ter conhecimento e publicações sobre o tema religiosidade/espiritualidade. Dos 15 especialistas que foram convidados, 5 contribuíram para o estudo em questão. Cada um dos cinco especialistas recebeu a versão original e a versão síntese em português junto com um instrumento específico para o registro das avaliações de equivalência cultural e conceitual. O instrumento continha as definições de cada tipo de equivalência, bem como as instruções para o seu julgamento.

Resultados

A seguir são apresentados no quadro 1 os itens originais da escala de necessidades espirituais e sua correspondente versão em português após as análises semântica, idiomática, cultural e conceitual por parte dos juízes especialistas.

Quadro 1 - Escala original de necessidades espirituais e versão em português.

\begin{tabular}{|c|c|}
\hline Itens originais & $\begin{array}{c}\text { Itens após análises } \\
\text { semântica, idiomática, cultural e conceitual }\end{array}$ \\
\hline 1. To review your life? & Rever a sua vida? \\
\hline 2. To be accepted as a person? & Ser aceito como pessoa? \\
\hline $\begin{array}{l}\text { 3. To participate in religious/spiritual services } \\
\text { or rituals? }\end{array}$ & Participar de serviços religioso-espirituais ou rituais? \\
\hline 4. To feel hopeful? & Sentir-se esperançoso? \\
\hline 5. To find meaning in suffering? & Encontrar um significado no sofrimento? \\
\hline $\begin{array}{l}\text { 6. To feel a sense of connection with the } \\
\text { world? }\end{array}$ & Sentir conexão com o mundo? \\
\hline 7. To be thankful or grateful? & Sentir-se grato ou agradecido? \\
\hline 8. To address issues about death and dying? & Tratar de questões sobre a morte e o morrer? \\
\hline 9. For companionship? & De companhia? \\
\hline 10. To have someone pray with or for you? & De alguém orar com ou por você? \\
\hline 11. For peace and contentment? & De paz e satisfação com a vida? \\
\hline 12. To experience or appreciate beauty? & De vivenciar ou apreciar o que é belo? \\
\hline 13. To find meaning and purpose in life? & Encontrar significado e propósito de vida? \\
\hline 14. For guidance from a higher power? & De direção de um poder superior? \\
\hline 15. To live an ethical/moral life? & De viver uma vida ética e moral? \\
\hline 16. To experience or appreciate music? & Ouvir ou apreciar música? \\
\hline $\begin{array}{l}\text { 17. To address concerns about life after } \\
\text { death? }\end{array}$ & Refletir sobre as preocupações com a vida após a morte? \\
\hline 18. To give or receive love? & Dar ou receber amor? \\
\hline 19. To participate in religious/spiritual rituals? & Participar em rituais religioso-espirituais? \\
\hline 20. To keep a positive outlook? & Manter uma perspectiva positiva? \\
\hline 21. To read spiritual or religious material? & Ler material espiritual ou religioso? \\
\hline 22. To forgive yourself and others? & Perdoar a si mesmo e aos outros? \\
\hline 23. For compassion and kindness? & De compaixão e bondade? \\
\hline $\begin{array}{l}\text { 24. To have a quiet space to mediate or } \\
\text { reflect? }\end{array}$ & Ter um espaço tranquilo para meditar ou refletir? \\
\hline 25. For respectful care to your bodily needs? & De cuidado respeitoso às suas necessidades corporais? \\
\hline 26. To experience or appreciate nature? & De vivenciar ou apreciar a natureza? \\
\hline $\begin{array}{l}\text { 27. To experience a sense of laughter and } \\
\text { humor? }\end{array}$ & Sentir-se bem-humorado? \\
\hline $\begin{array}{l}\text { 28. To make sense of why this crisis } \\
\text { happened to you? }\end{array}$ & Entender por que isso aconteceu com você? \\
\hline
\end{tabular}

Na sequência, apresentam-se nos próximos parágrafos os itens que após a tradução consensual receberam recomendações e sugestões dos juízes que participaram das análises semântica, idiomática, cultural e conceitual da escala Spiritual Needs Assessment Scale, assim, como os ajustes que foram efetuados nos respectivos itens.

Quanto à primeira questão da escala em estudo, os juízes sugeriram a substituição da palavra traduzida inicialmente para o português como "examinar" pela palavra rever, as quais levaram ao ajuste do referido item como apresentado a seguir:

Item 1: Examinar a sua vida?

Alterado para: rever a sua vida? 
No que concerne às questões números (6,7 e 27) os avaliadores sugeriram o ajuste dos itens do seguinte modo:

Item 6: Sentir uma sensação de conexão com o mundo?

Alterado para: Sentir conexão com o mundo?

Item 7: Ser grato ou agradecido?

Alterado para: Sentir-se grato ou agradecido?

Item 27: Experimentar uma sensação de riso e humor?

Alterado para: Sentir-se bem-humorado?

No item 11 foi mencionado que a palavra contentment ficava mais facilmente interpretada pelos pacientes como "satisfação". Dessa forma optou-se por realizar o ajuste da questão como sugerido.

Item 11: De paz e contentamento?

Alterado para: De paz e satisfação com a vida?

Outra sugestão recomendada diante das questões 12 e 26 da escala foi à substituição da palavra "experimentar" por "experienciar". Enfim, após discussão e análise das referidas questões os autores do estudo chegaram à conclusão que a palavra "vivenciar" seria a mais adequada no sentido de facilitar a interpretação e o entendimento dos itens mencionados. Dessa forma as questões foram ajustadas como se pode observar na sequência:

Item 12: De experimentar ou apreciar a beleza?

Alterado para: De vivenciar ou apreciar o que é belo?

Item 26: De experimentar ou apreciar a natureza?

Alterado para: De vivenciar ou apreciar a natureza?

As questões (16 e 17) também sofreram algumas modificações. Estes ajustes foram considerados pelos autores e alguns especialistas como necessários no sentido de facilitar a interpretação e entendimento das referidas questões.

Item 16: Experimentar ou apreciar música?

Alterado para: Ouvir ou apreciar música?

Item 17: Abordar preocupações sobre a vida após a morte?

Alterado para: Refletir sobre as preocupações com a vida após a morte?

$\mathrm{Na}$ última questão do instrumento, o significado da palavra inglesa crisis para o português foi comentada por um dos juízes convidados. Diante disso, após análise e discussão por parte dos pesquisadores chegou-se à conclusão que o respectivo item deveria ser alterado como demonstrado a seguir:

Item 28: Entender por que esta crise aconteceu com você? Alterado para: Entender por que isso aconteceu com você?

Finalmente após as contribuições e ajustes necessários obteve-se a versão da Spiritual Needs Assessment Scale traduzida transculturalmente para a língua portuguesa que recebeu 0 nome "Escala de Avaliação de Necessidades Espirituais" (Apêndice A). 
O processo de adaptação transcultural da Spiritual Needs Assessment Scale para a língua portuguesa originou a versão final da escala que recebeu o nome "Escala de Avaliação de Necessidades Espirituais". É importante ressaltar que, após esta fase fundamental, e para o uso do instrumento na prática clínica será realizado um novo estudo para avaliação da confiabilidade e validade da escala em questão. Enfim, vale ressaltar, que a utilização futura desse instrumento na prática clínica poderá ser um passo importante nessa área do conhecimento uma vez que poderá contribuir para a lacuna assistencial que existe nessa dimensão singular e de fundamental importância para o ser humano e com a qual os profissionais de saúde encontram dificuldades em abordar e intervir. Por último, como limitação do estudo cita-se o número total de juízes especialistas, embora os cinco participantes tenham contribuído de forma impar para o estudo, o número maior de juízes poderia ter levantado também outras questões, esclarecimentos e contribuições.

1. Tavares CQ, Valente CO, Cavalcanti APR, Carmos HO. Espiritualidade, religiosidade e saúde: velhos debates, novas perspectivas. Interações 2016;11(20):85-97.

2. Sweetman B. Religião: conceitos-chave em filosofia. Porto Alegre: Penso; 2013.

3. Borges MS, Santos MBC, Pinheiro TG. Representações sociais sobre religião e espiritualidade. Rev Bras Enferm 2015;68(4):609-16.

4. Dalgalarrondo P. Religião, psicopatologia e saúde mental. Porto Alegre: Artmed; 2008.

5. Koening H. Medicina, religião e saúde: o encontro da ciência e da espiritualidade. Traduzido por: Abreu I. Porto Alegre: L\&PM; 2012.

6. Lucchetti G, Lucchetti ALGL, Peres MF, Leão FC, Moreira-Almeida A, Koenig HG. Validação da versão brasileira da escala de religiosidade de Duke (DUREL). Rev Psiquiatr Clín 2010;39(4):130-5.

7. Larson DB, Swyers JP, McCullough ME. Scientific research on spirituality and health: a consensus report. National Institute for Healthcare Research. Rockville: The John M. Templeton Foudation; 1998.

8. Saad M, Masiero D, Battistella L. Espiritualidade baseada em evidências. Acta Fisiátr $2001 ; 8(3): 107-12$.

9. Reis NR, Soler ZASG. A espiritualidade na atenção à saúde: um tema atual para profissionais e usuários da saúde. Enfermagem Brasil 2016;15(5):236-7.

10. Astrow AB, Sharma RK, HuangY, Xu Y, Sulmasy DP. A Chinese Version of the Spiritual Needs Assessment for Patients Survey Instrument. J Palliat Med 2012;15(12):1297-315.

11. Koenig HG. Religion, spirituality, and medicine: research findings and implications for clinical practice. South Med J 2004;7(12):1194-1200.

12. Guimarães HP, Avezum A. O impacto da espiritualidade na saúde física. Rev Psiquiatr Clín 2007;34(1):88-94.

13. Esperandio MRG. Theology and the research on Spirituality and Health: a pilot study among health professionals and chaplains. Horizonte 2014;12(35):805-32.

14. Lucchetti G, Lucchetti ALG, Vallada H. Aferindo espiritualidade e religiosidade na pesquisa clínica: uma revisão sistemática dos instrumentos disponíveis no idioma Português. São Paulo Med J 2013;131(2):112-22.

15. Laurindo Filho J, Sá SPC, Chrizostimo MM, Oliveira FP, Pires BMFB, Lindolpho MC. A implicação da espiritualidade na saúde. Enfermagem Brasil 2016;15(5):280-284.

16. Flannelly KJ, Galek K, Flannelly LT. A test of the factor structure of the patient spiritual needs assessment scale. Holistic Nursing Practice 2006;20(4):187-190.

17. Galek K, Frannelly KJ, Vane A, Galek RM. Assessing a Patient's Spiritual Needs: A Comprehensive Instrument. Holistic Nursing Practice 2005;19(2):62-9.

18. Beaton DE, Bombardier C, Guillemin F, Ferraz MB. Guidelines for the process of crosscultural adaptation of self-report measures. Spine 2000;25(24):3186-91.

19. International Test Commission. International Guidelines on Test Adaptation. 2005. [citado 2016 Mai 12]. Disponível em: http://www.intestcom.org

20. Sireci SG, Faulkner-Bond M. Validity evidence based on test content. Psicothema 2014;26,100-7.

21. Pacico JC, Hutz CS. Validade. In: Hutz CS, Bandeira DR, Trentine CM. Psicometria. Porto Alegre: Artmed; 2015. 
Apêndice A - Escala de avaliação de necessidades espirituais.

Com que frequência você já experimentou cada uma dessas necessidades durante a sua estadia no hospital?

\begin{tabular}{|c|c|c|c|c|}
\hline Itens & Nunca & Raramente & $\begin{array}{c}\text { Com } \\
\text { Frequência } \\
\end{array}$ & $\begin{array}{l}\text { Com muita } \\
\text { Frequência }\end{array}$ \\
\hline 01. Rever a sua vida? & 1 & 2 & 3 & 4 \\
\hline 02. Ser aceito como pessoa? & 1 & 2 & 3 & 4 \\
\hline 03. Participar de serviços religioso-espirituais ou rituais? & 1 & 2 & 3 & 4 \\
\hline 04. Sentir-se esperançoso? & 1 & 2 & 3 & 4 \\
\hline 05. Encontrar um significado no sofrimento? & 1 & 2 & 3 & 4 \\
\hline 06. Sentir conexão com o mundo? & 1 & 2 & 3 & 4 \\
\hline 07. Sentir-se grato ou agradecido? & 1 & 2 & 3 & 4 \\
\hline 08. Tratar de questões sobre a morte e o morrer? & 1 & 2 & 3 & 4 \\
\hline 09. De companhia? & 1 & 2 & 3 & 4 \\
\hline 10. De alguém orar com ou por você? & 1 & 2 & 3 & 4 \\
\hline 11. De paz e satisfação com a vida? & 1 & 2 & 3 & 4 \\
\hline 12. De vivenciar ou apreciar o que é belo? & 1 & 2 & 3 & 4 \\
\hline 13. Encontrar significado e propósito de vida? & 1 & 2 & 3 & 4 \\
\hline 14. De direção de um poder superior? & 1 & 2 & 3 & 4 \\
\hline 15. Viver uma vida ética e moral? & 1 & 2 & 3 & 4 \\
\hline 16. Ouvir ou apreciar música? & 1 & 2 & 3 & 4 \\
\hline 17. Refletir sobre as preocupações com a vida após a morte? & 1 & 2 & 3 & 4 \\
\hline 18. Dar ou receber amor? & 1 & 2 & 3 & 4 \\
\hline 19. Participar em rituais religioso-espirituais? & 1 & 2 & 3 & 4 \\
\hline 20. Manter uma perspectiva positiva? & 1 & 2 & 3 & 4 \\
\hline 21. Ler material espiritual ou religioso? & 1 & 2 & 3 & 4 \\
\hline 22. Perdoar a si mesmo e aos outros? & 1 & 2 & 3 & 4 \\
\hline 23. De compaixão e bondade? & 1 & 2 & 3 & 4 \\
\hline 24. Ter um espaço tranquilo para meditar ou refletir? & 1 & 2 & 3 & 4 \\
\hline 25. De cuidado respeitoso às suas necessidades corporais? & 1 & 2 & 3 & 4 \\
\hline 26. De vivenciar ou apreciar a natureza? & 1 & 2 & 3 & 4 \\
\hline 27. De sentir-se bem-humorado? & 1 & 2 & 3 & 4 \\
\hline 28. Entender por que isso aconteceu com você? & 1 & 2 & 3 & 4 \\
\hline
\end{tabular}

